\title{
Wound dehiscence with nintedanib after cardiac surgery: A cautionary tale
}

\author{
Cheng He, MBBS, FRACS, ${ }^{\mathrm{a}}$ and Jaishankar Raman, MBBS, MMed, FRACS, PhD, ${ }^{\mathrm{a}, \mathrm{b}, \mathrm{c}}$ Melbourne, \\ Australia, and Portland, Ore
}

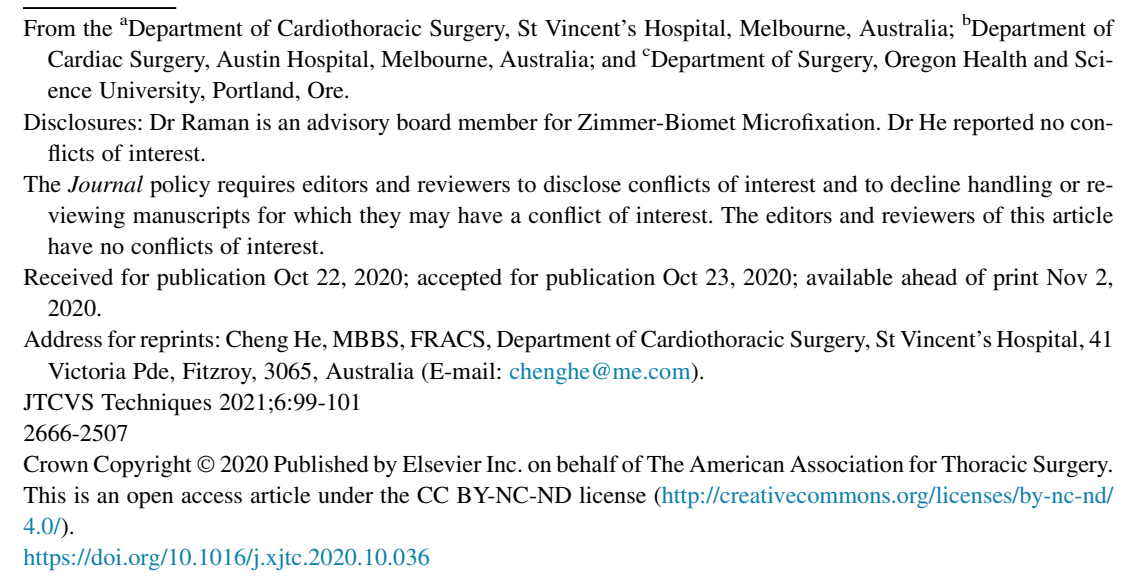

\section{CLINICAL SUMMARY}

A 67-year-old man (165 cm tall, weight $86 \mathrm{~kg}$ ) was transferred to our institution following unstable angina and a diagnosis of non-ST elevation myocardial infarction. Comorbidities included type 2 diabetes (HbA1c 7\%), hypertension, dyslipidemia, ex-smoker (30 pack-year history, quit 15 years ago) and newly diagnosed stable idiopathic pulmonary fibrosis (IPF) for which the antifibrotic medication, nintedanib, was commenced 5 months prior. The patient had minimal respiratory symptoms (no cough, sputum, nor infective exacerbations) and was regularly playing golf before admission. Coronary angiography revealed severe double-vessel disease, with a long ostial left anterior descending artery stenosis and a proximal left circumflex artery stenosis. Given the nature of the coronary disease and the patient's stable IPF, he was referred for inpatient surgical revascularisation following heart team discussion.

On-pump coronary artery bypass via median sternotomy with an in situ, pedicled left internal thoracic artery to the left anterior descending artery and radial artery to the second obtuse marginal artery was performed. The sternum was closed using 4 multifilament cables (Pioneer Surgical Technology Inc, Marquette, Mich) in figure-8 fashion. Nintedanib (150 mg twice daily) was continued preoperatively and restarted on postoperative day 2. Glycemic control was $<180 \mathrm{mg} / \mathrm{dL}$ perioperatively, with only one reading $>180 \mathrm{mg} / \mathrm{dL}$ on postoperative day 2 .

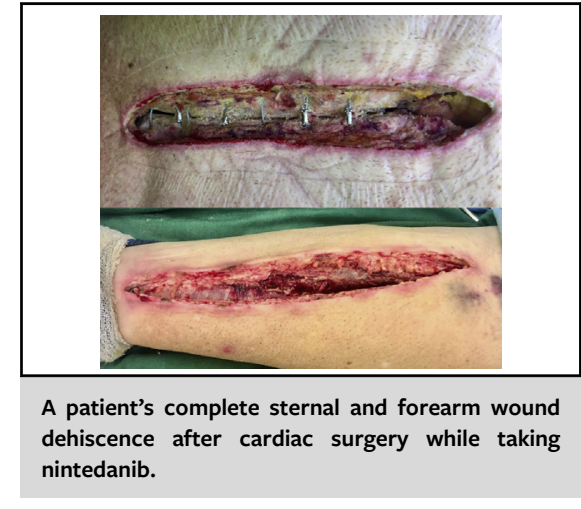

CENTRAL MESSAGE

Nintedanib, an antifibrotic agent used in pulmonary fibrosis, should be ceased perioperatively to prevent wound dehiscence after cardiac surgery.

See Commentaries on pages 102 and 104.

The patient was discharged, uneventfully, on postoperative day 6 . He represented postoperative week 4 with serous discharge from the sternotomy and left radial artery harvest wounds. The white cell count and inflammatory markers were within normal range. Blood cultures were negative. Operative exploration of both wounds revealed complete dehiscence of all soft-tissue layers and the absence of any fibrosis suggestive of healing (Figure 1, $A$ and $B$ ). Due to sternal instability, and the questionable sterility of the anterior mediastinum, sternal cables were removed to facilitate surgical washout. Sterile dehiscence was confirmed from both wounds, at all anatomical planes, after multiple negative wound swabs during vacuum-assisted dressing changes. Nintedanib was ceased following the initial operative washout findings.

The sternum was reapproximated this time using rigid closure technique, with 3 SternaLock plates (Zimmer Biomet, Jacksonville, Fla) (Figure 1, C). Closure with pectoralis major myocutaneous advancement flaps was performed to bridge the overlying soft tissue. The left forearm was closed using interrupted nylon sutures. The patient recovered well and at the 6-week follow-up, both wounds were 


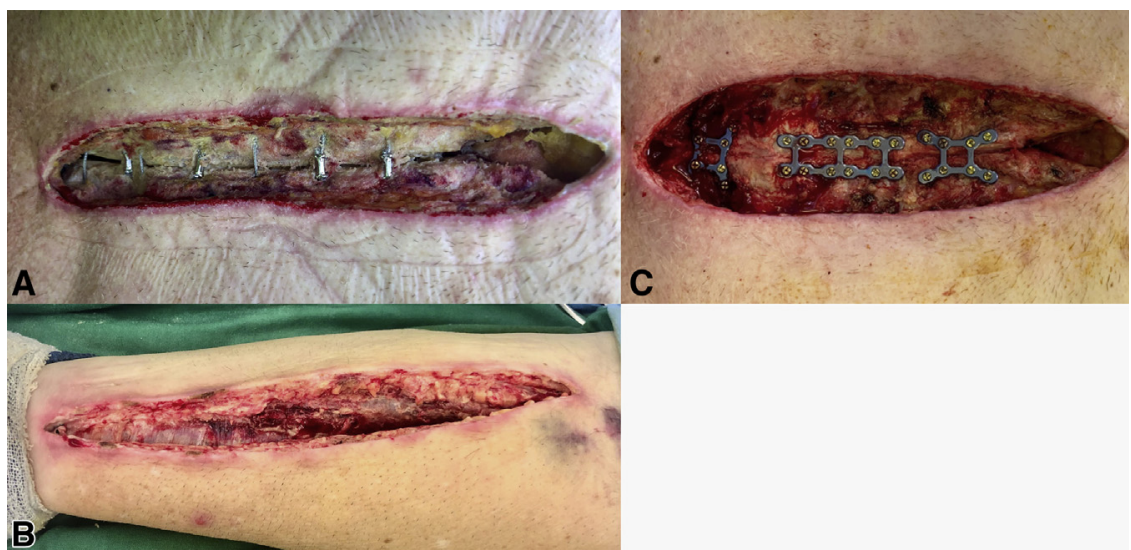

FIGURE 1. Surgical wounds 4 weeks postoperatively, at initial debridement, showing complete wound dehiscence at all surgical planes. A, Sternal wound with sternal cables in situ showing sternal nonunion. B, Left radial harvest site with no evidence of healing. C, Sternal plating following satisfactory wound debridements.

satisfactorily healed. Computed tomography demonstrated good sternal approximation (Figure 2). No further wound issues developed at last follow-up, 7 months post-closure, with nintedanib continued to be withheld in consultation with pulmonologists given stable pulmonary function testing and symptomatology.

\section{DISCUSSION}

Nintedanib, an antifibrotic medication, gained widespread approval for IPF treatment following the 2014 publication of a landmark trial. ${ }^{1}$ Concerns over postoperative wound complications, owing to the inhibition of multiple tyrosine kinases involved in wound healing, have been unfounded in observational studies. However, the literature is limited by a lack of statistical power, with the largest such report comprising 11 patients in the nintedanib arm. ${ }^{2}$ Further, published studies have focused exclusively on the lung transplantation setting. Because nintedanib administration is stopped after transplantation, postoperative healing is unlikely to be affected, given its short half-life (10 to 15 hours) ${ }^{3}$ and rapid clearance from the body. Like other tyrosine kinase inhibitors, the manufacturer's recommendation is for perioperative interruption and resumption of nintedanib once adequate wound healing has occurred. ${ }^{4}$

Sternal dehiscence is a dreaded complication of cardiac surgery. Numerous risk factors have been described, making exact causality difficult to define in every instance. Our patient had a concomitant complete forearm wound dehiscence, which insinuated a more systemic cause of wound-healing interference, making nintedanib a likely factor. In the absence of sternal infection, we utilized rigid plate sternal fixation due to its proven superior union outcomes in high-risk patients compared with traditional wire cerclage. ${ }^{5}$

Our case serves as a harsh lesson in the importance of apt perioperative assessment of patient medications and identification of their perioperative risks. Clearly, nintedanib should have been discontinued perioperatively, in accordance with the manufacturer's recommendations.

Informed consent was obtained from the patient for the publication of this case report. Authorization from St Vincent's Hospital (Melbourne) Human Research Ethics Committee was also obtained.

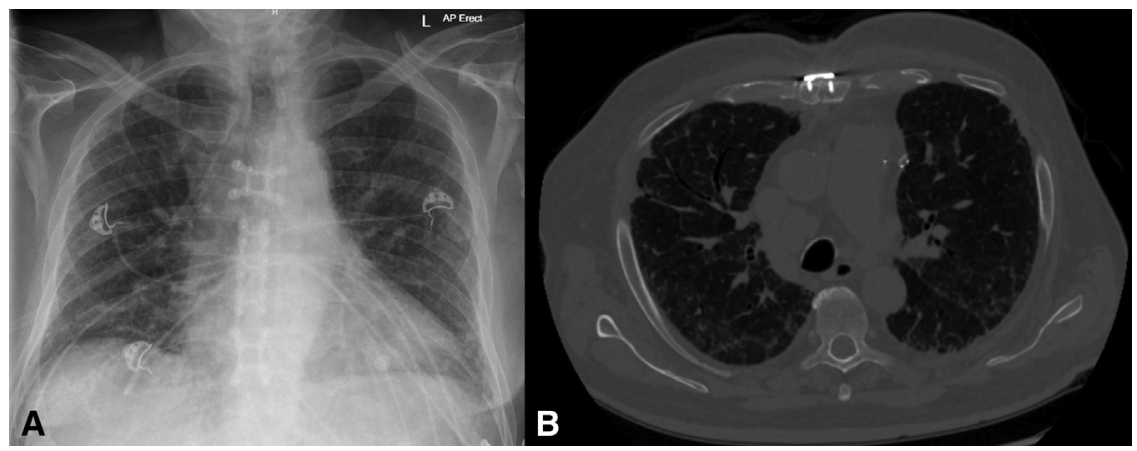

FIGURE 2. Imaging 3 months after rigid sternal plate closure. A, Chest radiograph showing the 3 SternaLock plates. B, Axial computed tomography slice through the superior sternum, showing good bony approximation. 


\section{References}

1. Richeldi L, Bois du RM, Raghu G, Azuma A, Brown K, Costabel U, et al. Efficacy and safety of nintedanib in idiopathic pulmonary fibrosis. N Engl J Med. 2014;370: 2071-82.

2. Mackintosh JA, Munsif M, Ranzenbacher L, Thomson C, Musk M, Snell G, et al. Risk of anastomotic dehiscence in patients with pulmonary fibrosis transplanted while receiving anti-fibrotics: experience of the Australian lung transplant collaborative. J Heart Lung Transplant. 2019; 38:553-9.
3. Wind S, Schmid U, Freiwald M, Marzin K, Lotz R, Ebner T, et al. Clinical pharmacokinetics and pharmacodynamics of nintedanib. Clin Pharmacokinet. 2019; 58:1131-47.

4. OFEV (Nintedanib) [product mongraph]. Boehringer Ingelheim (Canada) Ltd; 2020. Available at: https://www.boehringer-ingelheim.ca/sites/ca/files/ofevpme n 4.pdf. Accessed July 5, 2020.

5. Raman J, Lehmann S, Zehr K, De Guzman BJ, Aklog L, Garrett HE, et al. Sternal closure with rigid plate fixation versus wire closure: a randomized controlled multicenter trial. Ann Thorac Surg. 2012;94:1854-61. 